\title{
Vascular Smooth Muscle Cell Proliferation Is Influenced by let-7d MicroRNA and Its Interaction With KRAS
}

\author{
Man-Li Yu, MD; Jia-Feng Wang, MD; Guo-Kun Wang, MD; Xiao-Hua You, MD; \\ Xian-Xian Zhao, MD; Qing Jing, MD; Yong-Wen Qin, MD
}

\begin{abstract}
Background: Several microRNAs (miRNAs) have been reported to regulate cardiovascular biological and pathological processes through inhibiting the translation of certain RNA transcripts. However, little is known about the association between miRNAs and vascular smooth muscle cell (VSMC) proliferation. The aim was to investigate the role of miRNAs in VSMC growth and the potential mechanism.
\end{abstract}

\begin{abstract}
Methods and Results: Primary VSMCs were isolated from the medial layer of the thoracic aorta obtained from spontaneously hypertensive rats (SHR) and Wistar Kyoto rats (WKY). miRNA microarrays were used to analyze the difference in miRNA expression between VSMCs of SHR and WKY rats and were validated using TaqMan real-time PCR. Of the potentially related genes under the influence of let-7d identified through literature search, KRAS was verified by western blot and functionally analyzed using miRNA mimics transfection and analysis of transfectants by cell enumeration was made using CCK-8 and flow cytometric analysis of cell cycle progression. let-7d-transfected VSMCs from SHR, WKY and human coronary arteries expressed significantly lower amounts of KRAS protein, displayed reduced cell growth and led to a greater number of cells in the G1 phase than the G2/M phases of the cell cycle.
\end{abstract}

Conclusions: let-7d was significantly downregulated in VSMCs as an important regulator of cell proliferation. RAS might be involved in the proliferation regulation by let-7d. (Circ $J$ 2011; 75: 703-709)

Key Words: Genes; Hypertension; Vessels

$\mathbf{H}$ yperplasia and/or hypertrophy of the vascular smooth muscle cells (VSMCs) are central factors in cardiovascular diseases such as hypertension and atherosclerosis. Abnormal gene regulation is strongly implicated in the pathogenesis of hypertension, heart failure and arteriosclerosis characterized by abnormal proliferation and hypertrophy of the VSMCs, such as the KRAS/extracellular signal-regulated kinase (ERK) pathway, one of the most important pathways involved in VSMCs proliferation. ${ }^{1,2}$

MicroRNAs (miRNAs) are highly conserved endogenous short noncoding RNAs, regulating gene expression at the post-transcriptional level by inhibiting the translation of protein from mRNA or by promoting the degradation of mRNA. Their regulation is involved in many physiological and pathological conditions, including metabolism, differentiation development, apoptosis, proliferation and oncogenesis. ${ }^{3}$

Increasing evidence has demonstrated that several specific miRNAs function as a regulator of VSMC proliferation, apoptosis and differentiation by targeting the post-transcrip- tional expression of multiple genes. ${ }^{4-7}$ For example, it has been reported that Krüppel-like factor 5 (KLF5) is a target gene of miR-145 that is responsible for its regulatory effect on VSMC phenotypic modulation and VSMC proliferation. ${ }^{5}$ Others, such as mir221 and mir222, have been indicated to be novel regulators for VSMC proliferation and neointimal hyperplasia by inhibiting the expression of p27(Kip1) and p57(Kip2). ${ }^{7}$ These findings may represent new promising therapeutic targets for the treatment of a number of proliferative vascular diseases, such as atherosclerosis and hypertension. Genetically, spontaneously hypertensive rats (SHR) and Wistar Kyoto rats (WKY) strains share a common lineage, differing notably in their vascular phenotype. ${ }^{8}$ Since proliferation activity was much higher in VSMCs of SHR rats (SHR-VSMCs) than that of WKY rats (WKY-VSMCs), ${ }^{9}$ we speculated that miRNA expression profiles in SHRVSMCs might be responsible for the proliferation characteristics of SHR-VSMCs. Therefore, the present study was performed to screen the aberrantly expressed miRNAs in

Received April 26, 2010; revised manuscript received October 10, 2010; accepted November 5, 2010; released online January 24, 2011 Time for primary review: 10 days

Department of Cardiology (M.-L.Y., G.-K.W., X.-H.Y., X.-X.Z., Q.J., Y.-W.Q.), Department of Anesthesiology and Intensive Care Medicine (J.-F.W.), Changhai Hospital, The Second Military Medical University, Shanghai, China

The first two authors contributed equally to this work (M.-L.Y. and J.-F.W.).

Mailing address: Yong-Wen Qin, MD, Department of Cardiology, Changhai Hospital, The Second Military Medical University, 168

Changhai Road, Shanghai 200433, China. E-mail: qinyw_smmu@ @otmail.com

ISSN-1346-9843 doi:10.1253/circj.CJ-10-0393

All rights are reserved to the Japanese Circulation Society. For permissions, please e-mail: cj@j-circ.or.jp 

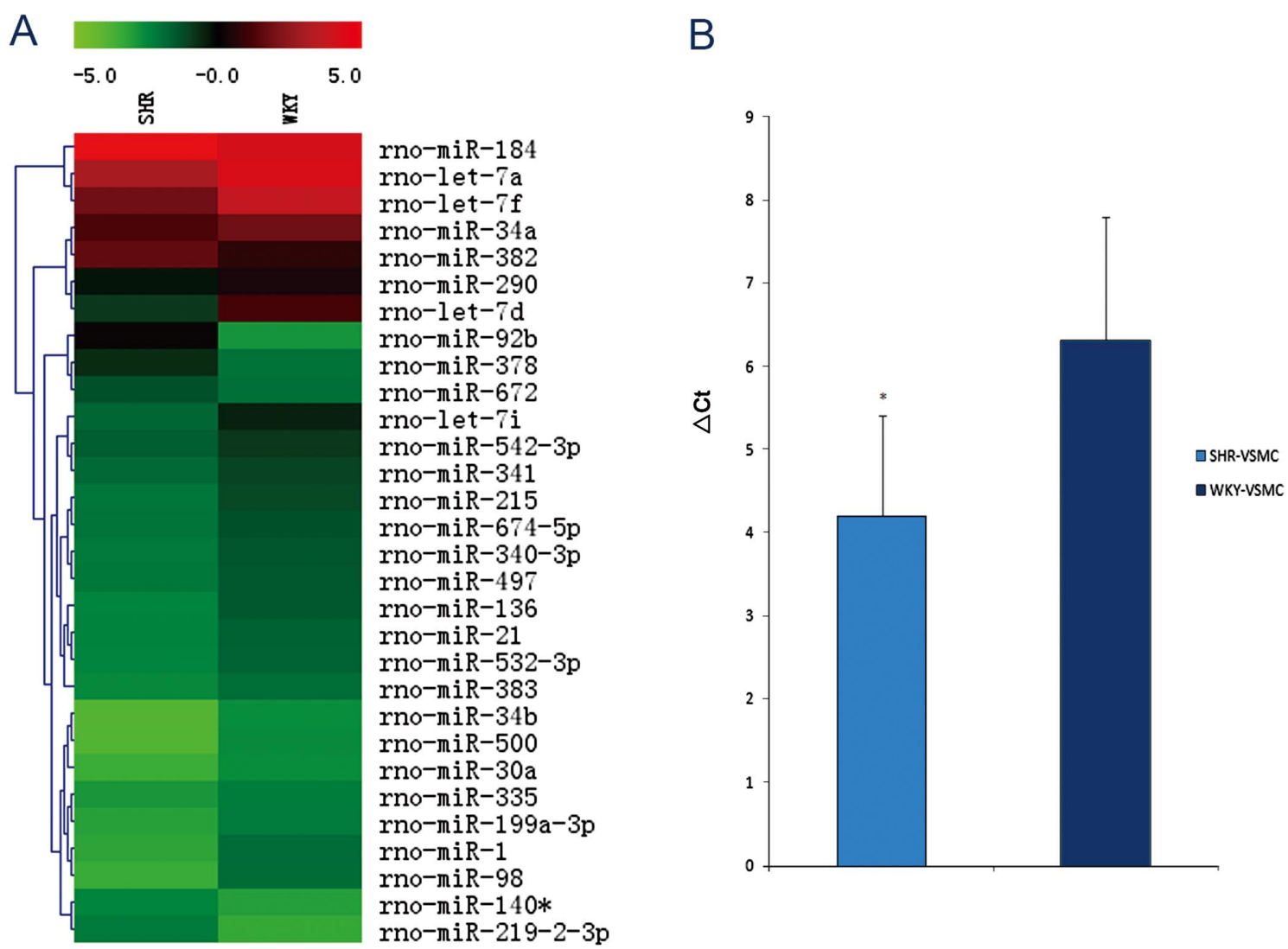

Figure 1. (A) Differential miRNA expression profiles from vascular smooth muscle cells (VSMCs) of SHR rats (SHR-VSMCs) and VSMCs of WKY rats (WKY-VSMCs) as shown in the form of heat maps Red, upregulation; green, downregulation; black, no change. (B) Expression level of let-7d in SHR- and WKY-VSMCs $(n=5)$. ${ }^{*} P<0.05$. SHR, spontaneously hypertensive rats; WKY, Wistar Kyoto rats.

SHR-VSMCs and also to investigate the possible related genes of these miRNAs.

\section{Methods}

\section{Cell Culture}

Rat aortic smooth muscle cells were isolated from the medial layer of the thoracic aorta obtained from female SHR and WKY rats (10 weeks old) and cultured in Dulbecco's modified Eagles medium (DMEM, Gibco BRL, USA) supplemented with $10 \%$ fetal bovine serum (FBS, Hyclone, USA), penicillin (10U/ml, Gibco BRL, USA), streptomycin (10 U/ml, Gibco BRL). The cells of passage 3-6 were used. The animal experiment was performed in accordance with the US National Institutes of Health guidelines and approved by the animal ethical committee of the Second Military Medical University. HCASMCs (Casecade, USA) were cultured in HCASMC PROLIF (M231/SMGS) (Invitrogen, USA) with the same treatment mentioned above.

\section{Analysis of the Expression of miRNAs by miRCURY LNA ${ }^{\mathrm{TM}}$ miRNA Arrays}

Total RNA of SHR-VSMCs and WKY-VSMCs were harvested using RNeasy mini kit (Qiagen, USA) according to the manufacturer's instructions. After RNA was quantitatively measured on the Nanodrop instrument and confirmed by gel electrophoresis, the samples were labeled using the miRCURYTM $\mathrm{Hy} 3^{\mathrm{TM}} / \mathrm{Hy} 5^{\mathrm{TM}}$ Power labeling kit and hybridized on the miRCURYTM LNA Array (v.11.0) (Exiqon, USA). Scanning was performed using the Axon GenePix 4000B microarray and the GenePix pro V6.0 was used to read and quantify the raw intensity of the image. The intensity of the green signal was calculated after background subtraction and averaged from 4 replicated spots of each probe on the same slide. The Median Normalization Method was used to obtain "Normalized Data" [Normalized Data $=$ (Foreground Background)/Median]. The median was the 50 percent quantile of the miRNA intensity that was greater than 50 in all samples after background correction. Fold-changes were calculated to identify the differentially expressed miRNAs.

\section{Candidate Validation by miRNA Real-Time Quantitative PCR}

Validation of candidate gene(s) from the miRNA microarray expression analysis was performed using real-time PCR with TaqMan ${ }^{\circledR}$ MicroRNA Assays (Applied Biosystems, USA). The PCR reaction consisted of 40 cycles $\left(95^{\circ} \mathrm{C}\right.$ for $15 \mathrm{~s}, 61^{\circ} \mathrm{C}$ for $15 \mathrm{~s}, 72^{\circ} \mathrm{C}$ for $\left.10 \mathrm{~s}\right)$ after an initial denaturation step $\left(95^{\circ} \mathrm{C}\right.$ for $3 \mathrm{~min}$ ). The PCR products were analyzed by electrophoresis on $2 \%$ agarose gels. GAPDH was used as an endogenous control. The results of real-time PCR were analyzed using the $\Delta \Delta \mathrm{Ct}$ method. ${ }^{10}$ 


\begin{tabular}{|c|c|c|c|c|c|c|c|c|}
\hline \multirow{2}{*}{ ID } & \multirow{2}{*}{ Name } & \multirow{2}{*}{$\frac{\text { Fold change }}{\text { SHR/WKY }}$} & \multicolumn{2}{|c|}{ Foreground } & \multicolumn{2}{|c|}{$\begin{array}{l}\text { Foreground- } \\
\text { background }\end{array}$} & \multicolumn{2}{|c|}{ Normalized } \\
\hline & & & SHR & WKY & SHR & WKY & SHR & WKY \\
\hline 17718 & miR-92b & 7.67 & 404 & 83 & 371 & 49 & 1.11 & 0.14 \\
\hline 11105 & miR-378 & 2.61 & 250 & 118 & 216 & 85 & 0.64 & 0.25 \\
\hline 42834 & miR-219-2-3p & 2.17 & 107 & 68 & 73 & 35 & 0.22 & 0.10 \\
\hline 14294 & miR-1 & 0.41 & 71 & 128 & 37 & 94 & 0.11 & 0.27 \\
\hline 27263 & let-7a & 0.42 & 279 & 6,799 & 2,756 & 6,765 & 8.20 & 19.59 \\
\hline 9938 & let-7i & 0.42 & 136 & 280 & 101 & 246 & 0.30 & 0.71 \\
\hline 29153 & miR-34b & 0.43 & 575 & 89 & 22 & 54 & 0.07 & 0.16 \\
\hline 42670 & miR-500 & 0.41 & 56 & 91 & 23 & 58 & 0.07 & 0.17 \\
\hline 46437 & let-7d & 0.25 & 215 & 784 & 181 & 750 & 0.54 & 2.17 \\
\hline 11182 & miR-98 & 0.35 & 65 & 126 & 32 & 93 & 0.10 & 0.27 \\
\hline 46435 & let-7f & 0.27 & 1,256 & 4,670 & 1,222 & 4,636 & 3.64 & 13.43 \\
\hline
\end{tabular}

Only miRNAs with changing fold higher than 2 or lower than 0.5 was showed.

\section{Transfection of let-7d miRNA Mimics}

The let-7d miRNA mimics and negative control (NC) (Genepharma, China) were diluted in the medium at a final concentration of $100 \mathrm{nmol} / \mathrm{L}$. Cells were incubated with let$7 \mathrm{~d}$ mimics and $\mathrm{NC}$ for $48 \mathrm{~h}$ using the fast transfection protocol recommended by Invitrogen. Efficiency of transfection was determined by comparing it against the NC provided by Genepharma.

\section{Cell Counting Kit-8 (CCK-8)}

Transfectants (SHR-VSMC/NC, SHR-VSMC/let-7d, WKYVSMC/NC, WKY-VSMC/let-7d, HCASMC/NC and HCASMC/let-7d) were resuspended in $200 \mu 1$ of cell culture medium and seeded in 96-well microtiter plates at $2.5 \times$ $10^{-4}$ cells $/ \mathrm{ml}$ and incubated for $48 \mathrm{~h}$ or $72 \mathrm{~h}$. Next, $10 \mu \mathrm{l}$ of CCK-8 reagent (Dojindo, Japan) was added to each well $2 \mathrm{~h}$ before the end of incubation. The optical density value (OD) of each sample was measured at a wavelength of $450 \mathrm{~nm}$ on a microplate reader (Multiskan MK3, Thermo Lab Systems). The results of cell viability measurement were expressed as the absorbance at OD450.

\section{Flow Cytometric Analysis}

Transfectants were typsinized, washed with PBS twice and then fixed with $70 \%$ ethanol on ice for $1 \mathrm{~h}$. The fixed cells were spun down, resuspended in PBS at $1 \times 10^{6}$ cells $/ \mathrm{ml}$. After incubation with ribonuclease (RNase A) at a final concentration of $3,000 \mathrm{units} / \mathrm{ml}$ at $37^{\circ} \mathrm{C}$ for $30 \mathrm{~min}$, trypsinized cells were filtered through a nylon mesh (BD Biosciences, USA). The cell suspension was stained with propidium idide before analysis on a FACSAria flow cytometer (BD Biosciences).

\section{Western Blot Analysis}

SHR-VSMCs, WKY-VSMCs and HCASMCs were trasnfected with let-7d miRNA mimics and NC. Cells transfected for $72 \mathrm{~h}$ were lysed in lysis buffer (Beyotime, China) for western blot on ice, and after being heated for $5 \mathrm{~min}$ at $95^{\circ} \mathrm{C}$, $20 \mathrm{ug}$ of denatured protein for each reaction was used to load a $10 \%$ polyacrylamide SDS $\left(\mathrm{H}_{2} \mathrm{O}, 1.5 \mathrm{~mol} / \mathrm{L}\right.$ Tris- $\mathrm{HCl}(\mathrm{pH}$ 8.8), $10 \%$ SDS, $30 \%$ Acrylamide/Bisacryl, $10 \%$ Am Persulfate, TEMED) for electrophoresis. Polyvinylidene difluoride membranes (Whatman, UK) were used for transfer by a wet transferring system (Bio-Rad, USA). The membrane was probed with primary antibodies against K-Ras-2A (diluted 1:100 in TBST, Santa Cruz, USA) at $4^{\circ} \mathrm{C}$ overnight, and with secondary HRP-conjugated anti-rabbit antibody (Santa Cruz) for $1 \mathrm{~h}$ at room temperature. After washing with $1 \times \mathrm{PBS}+0.1 \%$ Tween, the bands were detected by chemiluminescence (ECL detection kit, USA) and photographed with Kodak film (BioMax ${ }^{\circledR}$ Light-1, $18 * 24 \mathrm{~cm}$, USA). Twenty photos of each membrane were taken with an exposure interval of $15 \mathrm{~s}$. GAPDH was used as an endogenous protein for normalization. The ratio of KRAS/GAPDH was used for semi-quantification and comparison between different groups.

\section{Statistical Analysis}

All data were presented as mean \pm SD. One-way ANOVA was used to compare between samples. $\mathrm{P}<0.05$ was considered statistically significant. All experiments were performed at least 3 times.

\section{Results}

\section{miRNA Expression Profile Comparison Between SHR-VSMCs and WKY-VSMCs}

The differential expression of specific miRNA between SHRVSMCs and WKY-VSMCs suggest a potential difference in cell proliferation. Some miRNAs were decreased, such as let$7 \mathrm{~d}$ and $\mathrm{miR}-1$, which are shown as shades of green in the heat map. While some (eg, miR-92b, miR-378) were significantly increased as depicted by the shades of red in the heat map $(\mathrm{P}<0.05$, Figure 1A, Table). The entile miRNA expression profiles are provided in the supplemental file (Figure S1). To verify the accuracy of the microarray results, we made further identification using TaqMan real-time quantitative PCR. Results were validated using TaqMan real-time quantitative PCR, demonstrating a $\Delta \Delta \mathrm{Ct}$ of 2.1 cycles or 0.23 -fold change (equivalent to a 4.29-fold decrease) when comparing SHRVSMCs $(\Delta \mathrm{Ct}=4.2 \pm 1.2)$ with WKY-VSMC $(\Delta \mathrm{Ct}=6.3 \pm 1.5)$ $(\mathrm{n}=5, \mathrm{P}<0.05$, Figure 1B). Thus, let-7d was further studied to determine its potential biological function.

\section{Inhibition of KRAS Expression in VSMCs via let-7d Transfection}

According to the literature, let-7d reportedly downregulated KRAS in human as well as rat VSMCs. ${ }^{11,12}$ In the present study, SHR-VSMCs, WKY-VSMCs and HCASMCs were transfected with let-7d mimics and the rat/human KRAS protein levels were quantified by western blotting. Western blot analysis showed reduced expression of KRAS in the 3 
A

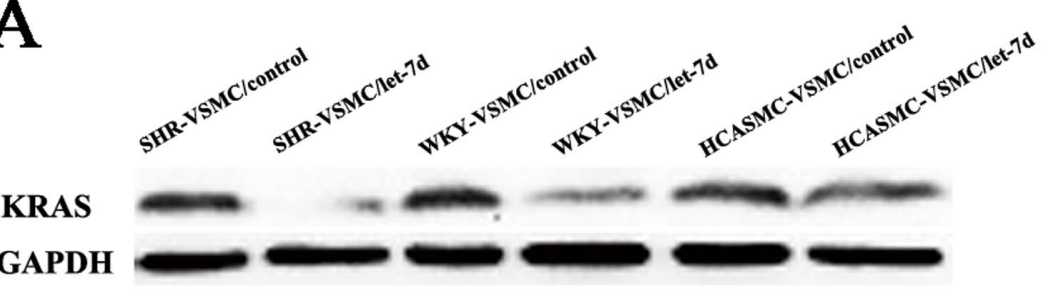

B

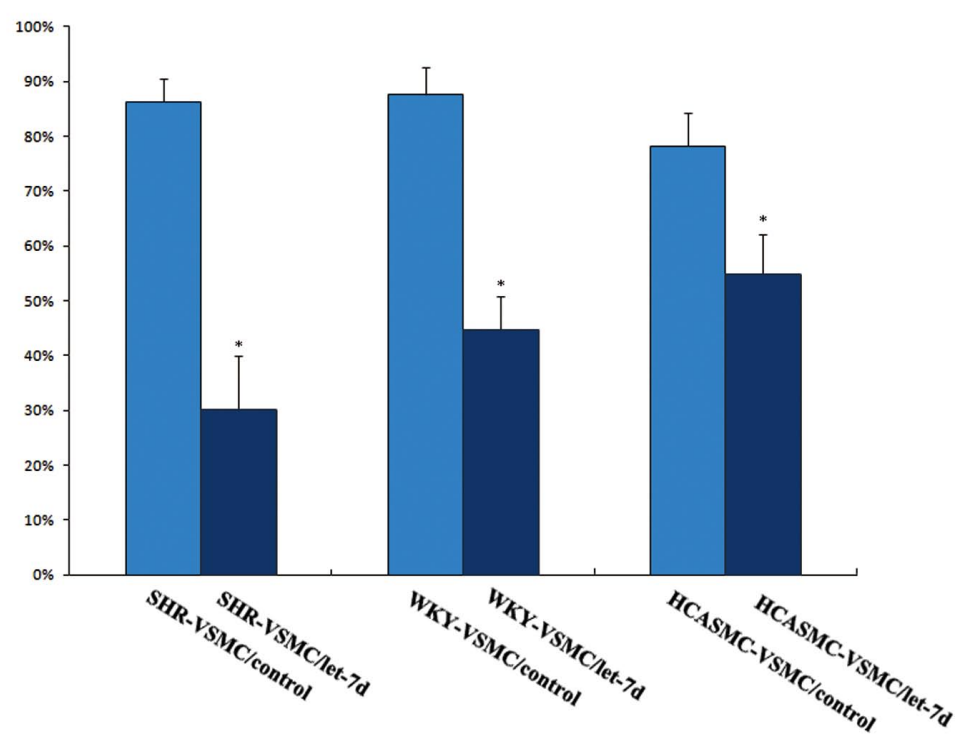

Figure 2. (A) Western blots of KRAS following let-7d mimics transfection of vascular smooth muscle cells (VSMCs) of SHR rats (SHR-VSMCs), VSMCs of WKY rats (WKY-VSMCs) and HCASMCs. GAPDH was used for protein level normalization. (B) Semi-quantitative analysis of western blot results. The $y$-axis represents the percentage of KRAS expression to control. $\mathrm{N}=4$ for each group. ${ }^{*} \mathrm{P}<0.01$ compared with each control. SHR, spontaneously hypertensive rats; WKY, Wistar Kyoto rats.
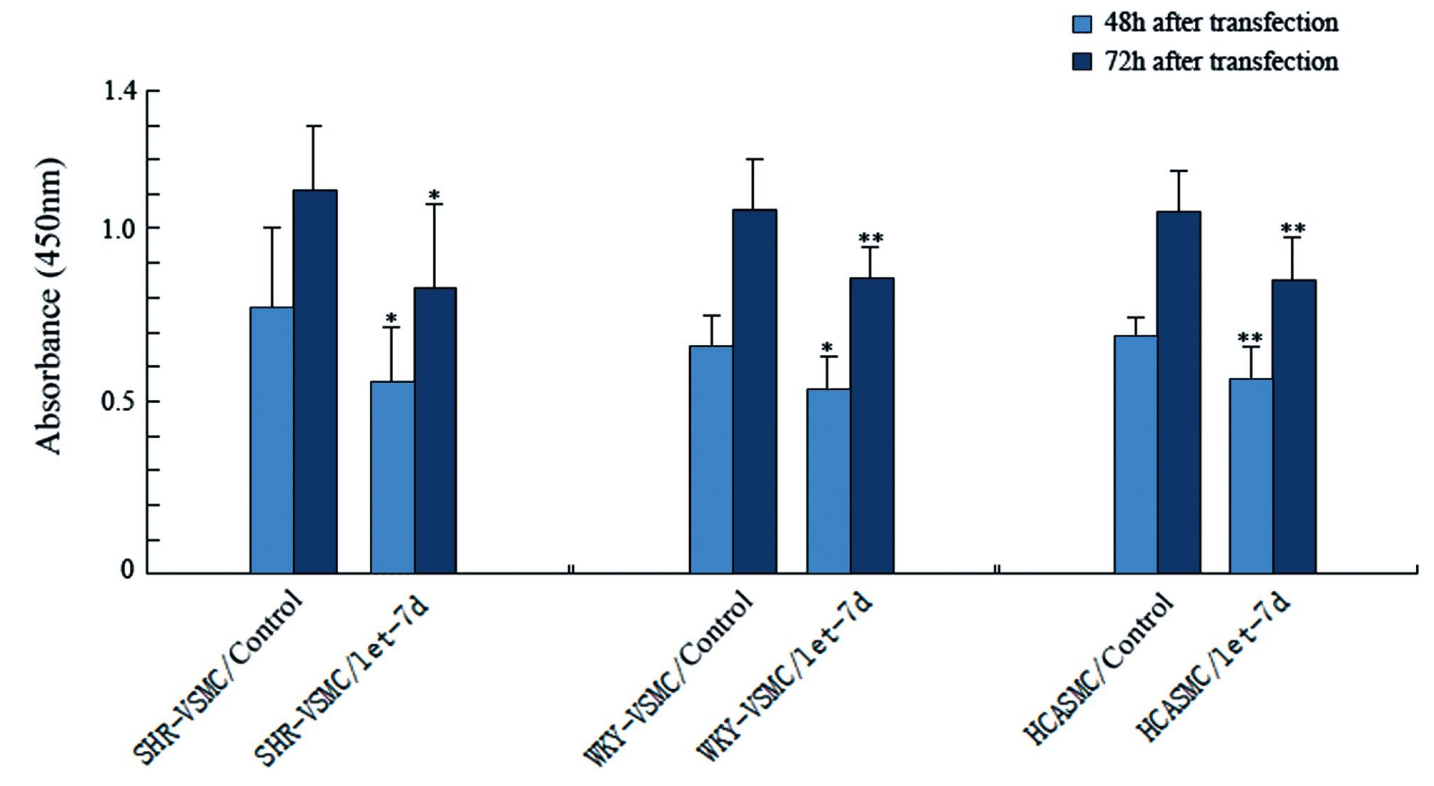

$\square 72 \mathrm{~h}$ after transfection

Figure 3. Effects of let-7d mimics on cell growth of vascular smooth muscle cells (VSMCs) of SHR rats (SHR-VSMCs), VSMCs of WKY rats (WKY-VSMCs) and HCASMCs. Cells in the logarithmic growth period were transfected by let-7d mimics or negative controls. The infected cells were reseeded to 96 -well plates. After incubation for $48 \mathrm{~h}$ and $72 \mathrm{~h}$, the proliferation of cells was detected using the CCK-8 assay. $N=6$ for each group. The values represent the means $\pm S D$. ${ }^{\star} P<0.05 ;{ }^{\star *} P<0.01$. SHR, spontaneously hypertensive rats; WKY, Wistar Kyoto rats. 

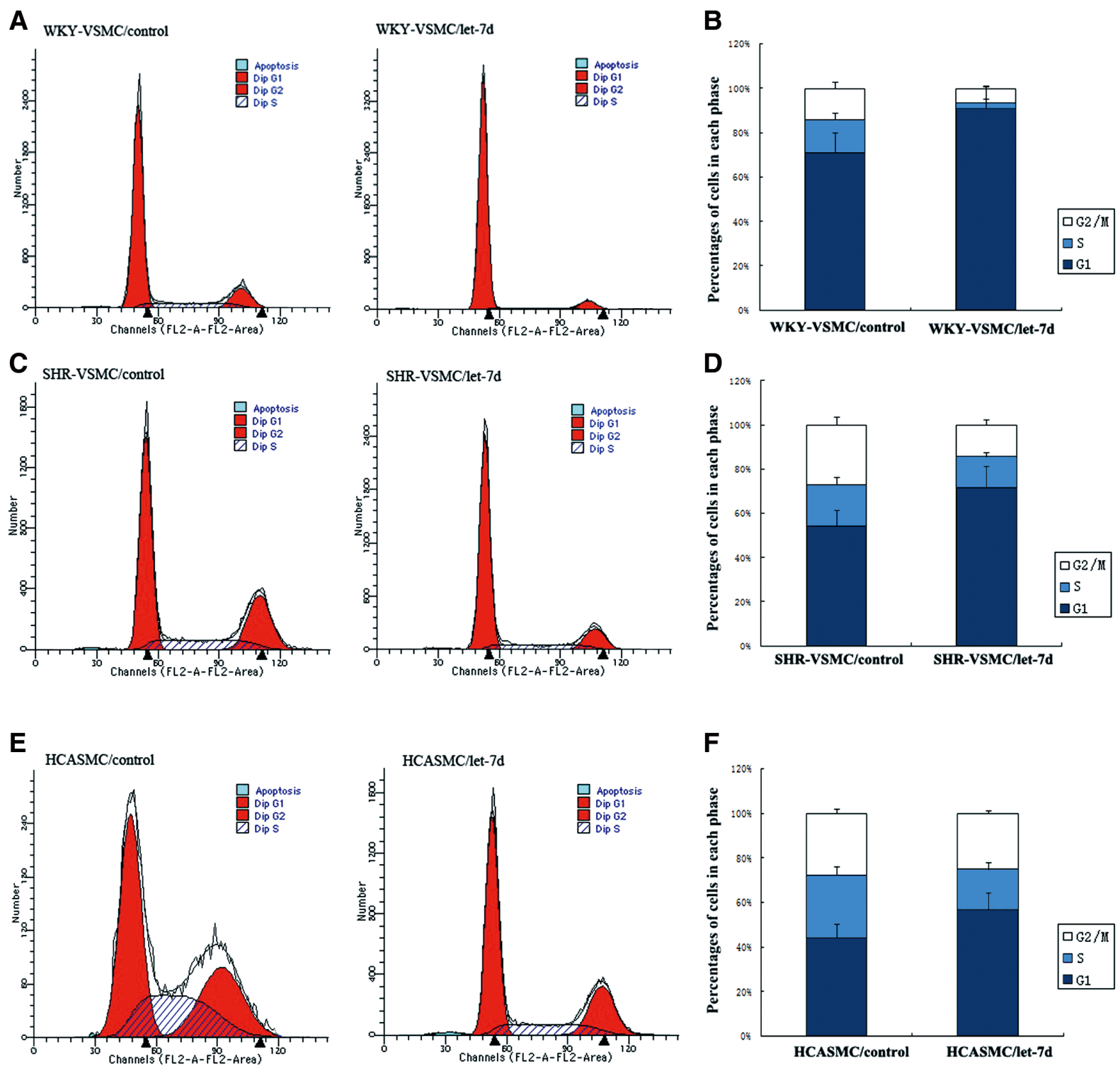

Figure 4. Effect of let-7d mimics on cell cycle of vascular smooth muscle cells (VSMCs) of SHR rats (SHR-VSMCs), VSMCs of WKY rats (WKY-VSMCs) and HCASMCs. (A) Flow cytometric analysis of SHR-VSMCs transfected with let-7d mimics or negative controls. (B) Percentages of cells at different phases in different groups of SHR-VSMCs. (C) Flow cytometric analysis of WKY-VSMCs transfected with let-7d mimics or negative controls. (D) Cell cycle progression of cells at different phases in different groups of WKY-VSMCs. (E) Flow cytometric analysis of HCAMSCs transfected with let-7d mimics or negative controls. (F) Percentages of cells at different phases in HCASMCs transfected by let-7d mimics or negative controls. $\mathrm{N}=4$ for each group. SHR, spontaneously hypertensive rats; WKY, Wistar Kyoto rats.

types of vascular cells transfected with let-7d (Figure 2).

\section{Effects of let-7d Overexpression on VSMC Proliferation}

SHR-VSMCs, WKY-VSMCs and HCAMSCs were transfected with let-7d miRNA mimics prior to the proliferation assay. CCK-8 assay was employed to measure cell proliferation. The results showed that exogenous expression of let-7d inhibited proliferation of VSMCs significantly (Figure 3). After incubation for $48 \mathrm{~h}$ and $72 \mathrm{~h}$, there were significant inhibitory effects in the transfected group compared with the controls. In SHR-VSMCs, WKY-VSMCs and HCASMCs, the inhibitory rates were $28.11 \%(\mathrm{P}<0.05), 18.77 \%(\mathrm{P}<0.05)$ and $17.32 \%(\mathrm{P}<0.01)$ after incubation for $48 \mathrm{~h}$, and $25.19 \%$ $(\mathrm{P}<0.05), 18.53 \%(\mathrm{P}<0.01)$ and $18.27 \%(\mathrm{P}<0.01)$ after incubation for $72 \mathrm{~h}$, respectively, which suggested overexpression of let-7d inhibited proliferation of VSMCs in vitro.

\section{Effects of let-7d Overexpression on the Cell Cycle of the VSMC}

Cell cycle analysis was performed to determine whether there was any cell cycle alteration in the 2 kinds of VSMCs mentioned above. The result showed that compared with control group the percentage of cells at the G1 phase was increased from $54.25 \%$ to $71.82 \%$ (SHR-VSMCs, $\mathrm{P}<0.01$ ), $71.23 \%$ to 
91.21\% (WKY-VSMCs, $\mathrm{P}<0.01$ ) and $44.13 \%$ to $56.81 \%$ (HCASMCs, $\mathrm{P}<0.01$ ), respectively, whereas the cells at the $\mathrm{S}$ phase decreased from $18.61 \%$ to $13.87 \%$ (SHR-VSMCs, $\mathrm{P}<0.01$ ), $14.58 \%$ to $2.27 \%$ (WKY-VSMCs, $\mathrm{P}<0.01$ ) and $28.43 \%$ to $18.21 \%$ (HCASMCs, $\mathrm{P}<0.05$ ) and the population of cells at the G2 phase decreased from $27.14 \%$ to $14.31 \%$ (SHR-VSMCs, $\mathrm{P}<0.01$ ), $14.19 \%$ to $6.51 \%$ (WKY-VSMCs, $\mathrm{P}<0.01 \%$ ) and $27.44 \%$ to $24.97 \%$ (HCASMCs, $\mathrm{P}<0.01$ ) (Figure 4). These results indicated that overexpression of let7d induced a G1 cell cycle arrest in VSMCs, and the percentage of the $\mathrm{S}$ and $\mathrm{G} 2 / \mathrm{M}$ phases were reduced significantly.

\section{Discussion}

miRNAs play an important and indispensable role in regulating cardiovascular biological and pathological processes. ${ }^{13}$ Various miRNAs have been found to be associated with VSMC proliferation and necrosis. Cheng et al found that miR-145 was a novel VSMC phenotypic marker and modulator that controlled vascular neointimal lesion formation. ${ }^{14}$ miR-221 and miR-222 were also identified to regulate VSMC proliferation and neointimal hyperplasia. ${ }^{7}$ Lin et al confirmed that miR-21 participated in $\mathrm{H}_{2} \mathrm{O}_{2}$-mediated gene regulation and injury response via its target gene Programmed Cell Death 4 (PDCD4) and AP-1 pathway with a protective effect against VSMC necrosis. ${ }^{15}$ Since proliferation activity was much higher in SHR-VSMCs, the aberrantly expressed miRNAs in SHR-VSMCs might be involved in VSMC proliferation regulation. Therefore, we made a thorough comparative screen using microarray analysis to identify the differentially expressed miRNAs that differed between SHRVSMCs and WKY-VSMCs, and pinpointed let-7d as a potential miRNA candidate responsible for the differing vascular phenotype.

The let-7 miRNA is among the founding members of the miRNA family and is highly conserved in invertebrates and vertebrates. Several studies have shown that let-7 regulates cell proliferation in cancer cells by inhibiting post-translation of RAS protein. ${ }^{11,16,17}$ However, its role in VSMC proliferation are currently unknown. In the present study, several miRNAs were expressed aberrantly in SHR-VSMCs compared with WKY-VSMCs, with supporting results also obtained from real-time quantitative PCR. These data may allow us to identify the miRNAs involved in VSMC proliferation and in the induction of vascular remodeling, atherosclerosis and hypertension. Our miRNA microarray data suggest that the expression levels of let-7d are significantly reduced in SHR-VSMCs, which exhibits higher growth rates compared with WKY-VSMCs. The results also indicate that let-7d might play an important role in the process of VSMC growth. As it has been confirmed that the KRAS gene is associated with the let-7 miRNA family in mammalian cells, ${ }^{11}$ we postulated that let-7d might regulate VSMC proliferation by inhibiting KRAS. In SHR-VSMCs, WKYVSMCs and HCASMCs transfected with let-7d mimics, both the expression of KRAS and cell proliferation rate significantly decreased, as demonstrated by the CCK-8 assay, flow cytometric analysis and western blot analysis. Therefore, these results strongly indicate that let-7d may be an important regulator of VSMC proliferation and that KRAS might be the key gene. Hu et al found that RAS expression in the artery of SHR was higher than that of WKY. ${ }^{18}$ However, in the present study, there was no significant difference between the RAS level in SHR-VSMCs and WKY-VSMCs, which could be the result of subculturing of the primary cells. ${ }^{19}$
Cells used for the microarray were at passage 3 , while cells of passage 5-6 were used to make the further functional study. Because the purpose of the present study was to investigate whether RAS expression and VSMC proliferation was regulated by let-7d, the difference in RAS expression between SHR and WKY-VSMCs was beyond the scope of the study.

VSMCs play a key role in fibrous cap formation and plaque stability in advanced atherosclerosis. ${ }^{20}$ They are the major producers of extracellular matrix within the vessel wall with the ability to modulate the type of matrix proteins produced in response to atherogenic stimuli. ${ }^{21}$ Increased proliferation and migration of VSMCs from media to intima of vessels resulted in VSMC accumulation and vascular remodeling. Thus, VSMC proliferation is a key event in the process of vascular diseases, such as hypertension and restenosis after balloon catheterization, ${ }^{22}$ which might be reversed by inhibiting VSMC proliferation. ${ }^{23}$ KRAS proteins are prototypical members of the superfamily of small GTPases. They transmit signals from cell surface receptors to a variety of effectors and thereby regulate pathways governing cell proliferation, differentiation and programmed cell death. Increased Ras/MEK/ERK signaling pathways in primary VSMC is implicated in the diagnosis and therapy of a variety of proliferative vascular diseases. Many efforts have been made in an attempt to develop Ras inhibitors as a therapeutic measure against vasculoproliferative diseases. In the present study, let-7d transfection resulted in a decrease of KRAS expression and an inhibition of VSMC proliferation; therefore, let-7d might be a potential therapeutic target of vasculoproliferative diseases.

\section{Conclusion}

In summary, the present study revealed that let-7d is significantly reduced in the VSMCs of the vascular disease strain, SHR, compared with the relatively healthy wild-type, WKY. Overexpression of let-7d led to a decrease in KRAS expression and VSMC proliferation. These results implicate let-7d as a miRNA regulator of VSMC proliferation via KRAS.

\section{Disclosure}

None declared.

\section{References}

1. Bornfeldt KE, Krebs EG. Crosstalk between protein kinase A and growth factor receptor signaling pathways in arterial smooth muscle. Cell Signal 1999; 11: 465-477.

2. Do e Z, Fukumoto Y, Takaki A, Tawara S, Ohashi J, Nakano M, et al. Evidence for Rho-kinase activation in patients with pulmonary arterial hypertension. Circ J 2009; 73: 1731 - 1739.

3. Cho WC. OncomiRs: The discovery and progress of microRNAs in cancers. Mol Cancer 2007; 6: 60-66.

4. Chan MC, Hilyard AC, Wu C, Davis BN, Hill NS, Lal A, et al. Molecular basis for antagonism between PDGF and the TGFbeta family of signalling pathways by control of miR-24 expression. EMBO J 2010; 29: 559-573.

5. Elia L, Quintavalle M, Zhang J, Contu R, Cossu L, Latronico MV, et al. The knockout of miR-143 and -145 alters smooth muscle cell maintenance and vascular homeostasis in mice: Correlates with human disease. Cell Death Differ 2009; 16: 1590-1598.

6. Zhang C. MicroRNA-145 in vascular smooth muscle cell biology: A new therapeutic target for vascular disease. Cell Cycle 2009; 8: $3469-3473$.

7. Liu X, Cheng Y, Zhang S, Lin Y, Yang J, Zhang C. A necessary role of miR-221 and miR-222 in vascular smooth muscle cell proliferation and neointimal hyperplasia. Circ Res 2009; 104: 476-487.

8. Louis WJ, Howes LG. Genealogy of the spontaneously hypertensive rat and Wistar-Kyoto rat strains: Implications for studies of inherited hypertension. J Cardiovasc Pharmacol 1990; 16(Suppl 7): 
S1-S5.

9. Uehara Y, Numabe A, Kawabata Y, Nagata T, Hirawa N, Ishimitsu $\mathrm{T}$, et al. Rapid smooth muscle cell growth and endogenous prostaglandin system in spontaneously hypertensive rats. Am J Hypertens 1991; 10: 806-814.

10. Livak KJ, Schmittgen TD. Analysis of relative gene expression data using real-time quantitative PCR and the 2(-Delta Delta C(T)) Method. Methods 2001; 25: 402-408.

11. Johnson SM, Grosshans H, Shingara J, Byrom M, Jarvis R, Cheng A, et al. RAS is regulated by the let-7 microRNA family. Cell 2005; 120: $635-647$.

12. Johnson CD, Esquela-Kerscher A, Stefani G, Byrom M, Kelnar K, Ovcharenko D, et al. The let-7 microRNA represses cell proliferation pathways in human cells. Cancer Res 2007; 67: 7713-7722.

13. Urbich C, Kuehbacher A, Dimmeler S. Role of microRNAs in vascular diseases, inflammation, and angiogenesis. Cardiovasc Res 2008; 79: 581-588.

14. Cheng Y, Liu X, Yang J, Lin Y, Xu DZ, Lu Q, et al. MicroRNA145 , a novel smooth muscle cell phenotypic marker and modulator, controls vascular neointimal lesion formation. Circ Res 2009; 105: $158-166$.

15. Lin Y, Liu X, Cheng Y, Yang J, Huo Y, Zhang C. Involvement of MicroRNAs in hydrogen peroxide-mediated gene regulation and cellular injury response in vascular smooth muscle cells. J Biol Chem 2009; 284: 7903-7913.

16. Kumar MS, Lu J, Mercer KL, Golub TR, Jacks T. Impaired microRNA processing enhances cellular transformation and tumorigenesis. Nat Genet 2007; 39: 673-677.
17. Büssing I, Slack FJ, Grosshans H. let-7 microRNAs in development, stem cells and cancer. Trends Mol Med 2008; 14: 400-409.

18. Hu WY, Han YJ, Gu LZ, Piano M, de Lanerolle P. Involvement of Ras-regulated myosin light chain phosphorylation in the captopril effects in spontaneously hypertensive rats. Am J Hypertens 2007; 20: $53-61$.

19. Passaquin AC, Schreier WA, de Vellis J. Gene expression in astrocytes is affected by subculture. Int J Dev Neurosci 1994; 12: $363-$ 372.

20. Doran AC, Meller N, McNamara CA. Role of smooth muscle cells in the initiation and early progression of atherosclerosis. Arterioscler Thromb Vasc Biol 2008; 28: 812-819.

21. Ottaviano FG, Handy DE, Loscalzo J. Redox regulation in the extracellular environment. Circ J 2008; 72: 1-16.

22. Inoue T, Node K. Molecular basis of restenosis and novel issues of drug-eluting stents. Circ J 2009; 73: 615-621.

23. Bornfeldt KE, Raines EW, Graves LM, Skinner MP, Krebs EG, Ross R. Platelet-derived growth factor: Distinct signal transduction pathways associated with migration versus proliferation. Ann NY Acad Sci 1995; 766: 416-430.

\section{Supplemental Files}

Supplemental File 1

Figure S1. The entile miRNA expression profiles in SHR-VSMC.

Please find supplemental file(s);

http://dx.doi.org/10.1253/circj.CJ-10-0393 